\title{
La inserción laboral tras los estudios universitarios. Los egresados de la Licenciatura en Publicidad y Relaciones Públicas
}

\section{The employability after college. Graduates of the Degree in Advertising and Public Relations}

Pedro Pablo Marín Dueñas. Universidad de Cádiz

Recibido: 22-XI-2012 - Aceptado: 27-II-2013

Resumen:

Existe un profundo interés en el seno de las instituciones universitarias por conocer qué ocurre con sus egresados cuando se introducen en el mercado laboral. El presente estudio se enmarca en este tipo de trabajos y se ha llevado a cabo siguiendo el método cuantitativo de la encuesta implementado a través de un cuestionario completado por egresados de tres promociones. En este artículo se exponen los principales aspectos metodológicos utilizados durante la investigación y se resumen los principales resultados obtenidos en dicho estudio que muestran datos positivos en relación con la inserción laboral de los egresados en Publicidad y Relaciones Públicas como el hecho de que el $82 \%$ de los egresados se encuentra trabajando en el momento de realización de la encuesta

Palabras Clave:

Egresados; inserción laboral; publicidad; universidad; comunicación

Abstract:

There is great interest within the universities to know what happens to their graduates when they introduced into the market work. This study is part of this type of work and was carried out using the quantitative method of structured survey. This article presents the main methodological aspects for research and summarizes the main results from this study show positive data regarding the employment of graduates in Advertising and Public Relations as the fact that $82 \%$ of graduates are currently working at the time of the survey.

Keywords:

Graduates; employability; advertising; higher education; communication 


\section{Introducción}

Desde hace varios años se ha venido fortaleciendo a nivel internacional la tendencia de la evaluación de la actividad universitaria, como una forma de rendición de cuentas a la sociedad y a los gobiernos.

En ese contexto de evaluación, el seguimiento de egresados es un asunto de vital importancia para las universidades, ya que el desempeño profesional y personal de los egresados permite establecer indicadores con respecto a la calidad y eficiencia de las instituciones de educación superior.

Hoy en día, las universidades deben analizar minuciosamente la inserción de los egresados en el mercado laboral para mejorar su oferta de enseñanza y formación, de ahí que los estudios sobre egresados traten de poner un poco de luz en el mundo de las relaciones entre el campo de la educación y el campo del trabajo. Relaciones que ya de por sí son muy complejas y cuyo estudio ha generado un amplio cuerpo teórico.

En los últimos años, las universidades e instituciones de educación superior en todo el mundo han empezado a centrarse en el aseguramiento de la calidad para satisfacer las necesidades tanto de sus estudiantes como de la sociedad con respecto al mercado laboral. Por lo tanto, el conocimiento bien establecido de las fuerzas y las debilidades de sus programas de estudio es esencial para la gestión de la calidad. Al mismo tiempo, se exige cada vez más a las instituciones de educación superior que se responsabilicen de su trabajo. Por consiguiente, se mide el éxito de las universidades basado principalmente en el resultado de sus estudiantes con respecto a su situación laboral y su compromiso social.

Existe, en el mundo académico, un interés creciente por conocer las relaciones existentes entre la educación superior y el entorno del trabajo y que se ha convertido, en las últimas décadas, en uno de los temas de estudio fundamentales en la agenda de las universidades e instituciones educativas. Estos trabajos lo que tratan es de entender los caracteres socioeconómicos, las trayectorias laborales, educativas y los aspectos sociales de los que han sido sus alumnos, y que ahora son profesionales en los distintos ámbitos del mundo laboral.

Las instituciones de educación superior intentan con estos estudios comprender la calidad de sus programas educativos, y lo hacen investigando sobre el desempeño laboral de sus egresados y el grado de inserción laboral de los mismos. De esta forma se intenta establecer el éxito o fracaso de los actuales planes de estudio de sus titulaciones. Y los estudios de seguimiento de egresados constituyen una manera de realizar esta medición. Este tipo de estudios va a permitir localizar deficiencias en los programas educativos y, de esta forma, planificar los futuros planes de estudio que realmente den respuesta a las necesidades del mercado laboral.

Es importante para las instituciones educativas conocer y valorar el desempeño profesional de sus titulados, conocer de primera mano si la educación impartida en sus titulaciones fue de calidad y les brindó a los graduados las oportunidades de desarrollo profesional que ellos buscaban al comenzar estos estudios. En definitiva, se trata de responder a la 
siguiente pregunta: ¿̇están los estudiantes preparados para incorporarse al mercado laboral con la formación recibida en la universidad?

Este trabajo trata de evaluar el producto final de la universidad (el egresado) que permita, a su vez, evaluar la titulación impartida para, de esta forma, mejorar de cara a la formación de futuros alumnos y de paso, mejorar la comunicación entre la universidad y los titulados.

Este estudio presenta los resultados de una investigación desarrollada durante el curso académico 2011-2012 realizada a los egresados de la Licenciatura en Publicidad y Relaciones Públicas de la Universidad de Cádiz de los cursos académicos 2005-2007, 2006-2008 y 2007-2009 con la finalidad de tener un primer acercamiento a su primera experiencia laboral y su situación laboral actual así como identificar cuál ha sido el impacto que esta Licenciatura tuvo para su desarrollo profesional.

Y, por tanto, este es el objetivo primordial de nuestro estudio. Conocer cómo se ha desarrollado la inserción laboral de los egresados de tres promociones de la Licenciatura en Publicidad y Relaciones Públicas y extraer los principales resultados que nos ayuden a determinar el grado de inserción laboral de los titulados.

\section{Estado de la cuestión}

\subsection{Los estudios de publicidad en España}

Según los últimos datos disponibles del Servicio de Estadística Universitaria del Ministerio de Educación, referidos al curso académico 2011-2012, había en España cuarenta y siete universidades que incorporaban en su oferta alguna de las tres titulaciones en Comunicación (periodismo, comunicación audiovisual y publicidad y relaciones públicas): veintinueve públicas y dieciocho privadas: Según datos del Ministerio de Educación, de las diecisiete Comunidades Autónomas en las que se divide la geografía española, trece cuentan con universidades que imparten alguna de las tres titulaciones en Comunicación vigentes en España: Cataluña es la Comunidad Autónoma con mayor oferta en estudios de Comunicación, ya que existen once universidades donde se imparte alguna de las tres carreras, seguida de Madrid, que posee ocho universidades con alguna de estas tres titulaciones y el tercer lugar lo ocupan Valencia y Castilla-León, donde los estudiantes pueden escoger entre seis universidades diferentes para realizar sus estudios en alguna de estas disciplinas. En el caso concreto de Andalucía existen cuatro, una de ellas la Universidad de Cádiz, en la que se enmarca el presente estudio. El resto de centros que ofrecen estos estudios se distribuyen de la siguiente manera: en Galicia tres y en Murcia y en el País Vasco dos. Navarra, Extremadura, Canarias, Baleares y Aragón poseen sólo una universidad que oferta estudios en Comunicación. Las cuatro Comunidades Autónomas en las que no existe esta oferta, son las siguientes: Asturias, Cantabria, Castilla-La Mancha y La Rioja. 
En el caso concreto de la titulación de Publicidad y Relaciones Públicas puede estudiarse en treinta y dos de las 47 universidades que ofertan estudios de comunicación. De estas treinta y dos, 16 son de titularidad pública y 16 de titularidad privada. La Comunidad Autónoma con mayor peso específico en los estudios de Publicidad y Relaciones Públicas en nuestro país es Cataluña, que concentra nada menos que el 29\% de las instituciones universitarias que ofertan esta titulación en España, con un total de nueve centros. En segundo lugar encontramos a Madrid, que alberga el 23\%, con un total de siete. El tercer puesto está copado por tres Comunidades Autónomas: Valencia, Castilla León y Andalucía que aglutinan cada una el $10 \%$ de los centros que ofertan esta titulación en nuestro país. Es necesario resaltar el hecho de que de las trece autonomías en las que se puede estudiar alguna de las tres carreras objeto de estudio, Publicidad y Relaciones Públicas sólo esta disponible en diez de ellas, faltando su implantación en Canarias, Baleares y Extremadura.

En lo tocante al número de alumnos matriculados, los últimos datos de los que se disponen, apuntan a que en el curso académico 2008-2009, hubo más de cuarenta y ocho mil alumnos matriculados en las tres disciplinas (contando con los más de dos mil alumnos matriculados en alguno de los títulos de grado que ya se están impartiendo en nuestro país). Según datos facilitados por el Ministerio de Educación, en Periodismo se matricularon en total 17.586 alumnos, 16.566 lo hicieron en Publicidad y Relaciones Públicas y 12.486 se apuntaron en Comunicación Audiovisual. En cuanto al número de licenciados, en el último curso académico del que se disponen datos (2006/2007) hubo un total de 6.773 graduados en alguna de las tres titulaciones en Comunicación que se ofertan en nuestro país. La carrera en la que más licenciados hubo fue Periodismo, con 2.601. En segundo lugar encontramos Publicidad y Relaciones Públicas con un total de 2.204 graduados, y por último Comunicación Audiovisual con 1.968.

Todos estos datos ponen de manifiesto la enorme importancia que están adquiriendo los estudios de Comunicación, para los que la demanda aumenta cada año frente a la tónica general que están experimentando la mayoría de las titulaciones en las que ha descendido, lo que conlleva una paulatina pérdida de alumnos. El principal problema que plantea el enorme crecimiento que experimentan estas titulaciones es la inserción en el mercado laboral de los titulados, ya que cada vez son más y es necesario valorar si pueden ser absorbidos por el mismo, porque de lo contrario estaríamos ante un grave problema: se incrementa el número de centros en los que se puede estudiar alguna de las disciplinas en Comunicación por su elevada demanda, y sin embargo el mercado laboral no es capaz de absorber a los licenciados, lo que conllevaría elevados índices de paro entre los titulados en alguna de estas especialidades o su inserción en trabajos no relacionados con el área para la que se han preparado durante años.

\subsection{La situación del mercado de trabajo en el sector de la Publicidad y las RRPP}

El Instituto Nacional de Empleo (INEM), a través de su Observatorio de las Ocupaciones del Servicio Público de Empleo Estatal, ofrece los datos más actuales en cuanto al nivel de ocupación y desempleo de los licenciados en Publicidad y Relaciones Públicas. 
Según el último informe disponible, de octubre de 2012, los Servicios Públicos de Empleo han registrado un total de 5371 contratos acumulados durante el año a un total de 3296 personas demandantes de empleo en esta titulación. En el mes concreto de octubre, las cifras registradas son de 610 contratos a 539 personas, de los que 544 han sido de duración temporal y 66 han sido de jornada completa. Por sexos, 463 de esos contratos han sido a mujeres y 147 a hombres y por tramos de edad, la mayoría de los contratos, 469, han sido a personas con entre 25 y 39 años. Con más de 40 años han sido contratadas 81 personas y con menos de 25 un total de 60 jóvenes han encontrado un empleo.

En total, en este mes, se han registrado 4775 demandantes de empleo, de los que 3849 son parados, lo que supone un incremento de 112 parados con respecto al mes anterior. De estos parados, 3661 han trabajado anteriormente. Si desagregamos estos datos, por sexos, 2891 parados son mujeres por 958 hombres. Con respecto a la edad, 1085 parados tienen ente 25 y 29 años, 1640 entre 30 y 39. Con más de 40 años encontramos a 873 personas y con menos de 25 a 251.

Con relación a los puestos contratados, la mayoría de las ocupaciones no guardan relación con la titulación y los intereses de los titulados. Empleados administrativos sin tareas de atención al público, recepcionistas (excepto de hoteles), teleoperadores, empleados administrativos con tareas de atención al público, camareros asalariados, vendedores en tiendas y almacenes, promotores de venta o azafatos de tierra son algunas de las áreas de actividad en las que se han empleado los licenciados en Publicidad en el mes de octubre.

Podríamos, con estos datos, atestiguar que aunque los titulados en Publicidad y Relaciones Públicas se encuentran en la actualidad trabajando, una parte importante no lo hace en aquello para lo que fue formado en la Universidad, de ahí que nos cuestionemos si este problema es debido a los planes de estudio de las facultades que no se adecuan a las demandas del mercado laboral y no les garantizan a los egresados una salida profesional relacionada con aquello que decidieron estudiar.

\section{Marco teórico}

Para lograr una educación de excelencia que lleve a formar a los profesionales del siglo XXI, es necesario fortalecer la cultura de evaluación del proceso educativo por medio de instrumentos y mecanismos que permitan retroalimentar la calidad de este proceso. Se transita sobre el sentido de la educación hacia una perspectiva productivista que coloca al empleo como punto central del fin educativo. Capacitar para el empleo se convierte en el eje de la mayor parte del debate pedagógico de este siglo (Hernández et al., 2006). Teniendo en cuenta esto, la evaluación del sistema educativo universitario se debe enfocar en la obtención de un empleo. Es decir, se puede medir la eficiencia de las instituciones de educación superior desde la perspectiva del empleo de sus egresados (Barajas, 2000). 
Para analizar esta relación entre educación y empleo es necesario fundamentar las principales teorías que explican los vínculos entre educación y el empleo de las personas. En este contexto, es importante señalar que a través de la historia se han desarrollado diversas teorías que tratan de explicar esta relación, entre las que podemos destacar la Teoría del Capital Humano, la Teoría de Bien Posicional, la Teoría de la Devaluación de los Certificados o la emergente Teoría del Enfoque Alternativo, entre otras. En este trabajo de investigación sólo se va a realizar un breve repaso a los fundamentos principales de dichas teorías.

Si bien, como se reconoce, existen en el ámbito del conocimiento diversas teorías que intentan explicar las relaciones entre el mundo de la educación superior y el mundo del trabajo, podríamos afirmar que los estudios de egresados se sustentan principalmente en la Teoría del Capital Humano. Dicha teoría, que sentó las bases de la Economía de la Educación, según Gleizes (2000), se entiende "como el conjunto de las capacidades productivas que un individuo adquiere por acumulación de conocimientos generales o específicos... La noción de capital humano expresa la idea de un stock inmaterial imputado a una persona que puede ser acumulado, usarse". De ahí que la Teoría del Capital Humano, es de suma importancia para entender el comportamiento que manifiestan los egresados de una institución cuando se enfrentan a la realidad de las organizaciones.

Los antecedentes a esta teoría los podemos encontrar en las obras de Adan Smith y Stuart Mill, que ponen el acento en la educación como pieza clave en el incremento productivo de los trabajadores, pero no será hasta la segunda mitad del siglo XX cuando se empiece a estudiar de forma notable la influencia que la educación tiene para el desarrollo económico de los países. Pero ya a finales del siglo XVIII se empieza a gestar la idea de que el nivel de educación de los individuos de una u otra forma incidía en los resultados en términos económicos; se decía que un hombre que había sido educado o instruido, al utilizar dicha educación o habilidad aprendida obtendría ingresos a través de los cuales recuperaría todos los gastos que hizo para obtener dicha educación. De igual manera, una explicación para la diferenciación de los niveles salariales obtenidos y de los cargos de trabajo ocupados entre los individuos, se explicaron bajo el principio del nivel de escolaridad o educación. En esencia, la idea básica era considerar a la educación y la formación como inversiones que realizan individuos racionales, con el fin de incrementar su eficiencia productiva y sus ingresos.

Ya a mediados del siglo XX se retoma esta idea de considerar a la educación y a la formación como una inversión. Según esta visión, se puede considerar a Theodore Schultz como el iniciador de esta teoría, para quien la clave está en considerar a la educación, o capital humano, como un bien de inversión (Schultz, 1968). Con su obra "Invertir en el hombre: la visión de un economista", trata de justificar que la teoría del capital humano reduzca o compare al individuo a una máquina Siguiendo esta teoría se puede entender que si el incremento de la inversión en educación contribuye a la mejora de la economía, los gobiernos, a través de la satisfacción de las demandas educativas, contribuirán al crecimiento económico. Esta inversión, por tanto, genera beneficios para los individuos, que ven aumentadas sus posibilidades de encontrar empleo, y de esta manera, incrementar sus ingresos, devolviendo además a la sociedad esa inversión. 
Por otro lado, la obra "Measuring the Contribution of Education (and the residual) to economic growth", presenta pruebas de que existe una correlación positiva entre el incremento en gasto educativo y el incremento del producto nacional bruto. En esta obra, además, se presentan como argumento que la inversión en educación es preferible a las inversiones en capital físico. Un tercer trabajo que sustenta a esta Teoría del Capital Humano es la obra "Human Capital" de Gary Becker a partir de la cual se estructuró el denominado Modelo simple de capital humano, modelo que sugiere que la educación es alguna forma de inversión que afecta positivamente a variables como el ingreso, el empleo, el crecimiento económico y la equidad social.

Otros autores como Harbison y Myers (1964) llegaron a afirmar que "la educación media y superior per cápita se encontraba altamente relacionada con el ingreso per cápita de un país” y que "los fenómenos del ingreso y del desempleo están asociados a las diferencias en los niveles educativos". Esta teoría tuvo grandes consecuencias en los países capitalistas, provocando la expansión de los sistemas educativos, incrementándose el gasto público en educación.

Como no podía ser de otra forma, esta teoría ha recibido muchas críticas. Así, por ejemplo, Blaug (1978) y Duncan (en Carnoy, 1982) afirman que el nivel socioeconómico de los padres influye en la formación recibida y que por tanto, los ingresos familiares se convierten en un factor determinante para la educación. Es, por tanto, la educación de élite la que facilita el acceso a los mejores empleos y no la productividad del trabajador.

Otros autores afirman que son las características del empleo las que determinan la productividad de las personas y no al revés. No es la educación previa el hecho relevante de cara a aumentar la productividad sino que las habilidades se aprenden a través del entrenamiento y la capacitación en el propio puesto de trabajo, y de ahí deriva la productividad. Por tanto, no se contrata al mejor formado, sino al más apto para ser capacitado (Thurow y Lucas en Navarro, 1998).

Sewell y Hauser (en González, 1999), por su parte, consideran que los logros de los padres explican el nivel ocupacional de una persona y que los logros educativos de los hijos vienen explicados por el estatus ocupacional de los padres. Es decir, los jóvenes encuentran trabajos en función de la clase social de los padres, clase social que viene determinada por los ingresos, la ocupación y los estudios, y que lo salarios de estos jóvenes viene determinado también por este aspecto. Por tanto, la educación no es un factor determinante de la productividad, sino simplemente de legitimación social.

La Teoría de la Educación como bien posicional gira en este mismo sentido. Esta teoría, según Anuies (1998) consiste en: "Explicar la heterogeneidad escolar de las personas que se desempeñan en las ocupaciones existentes en los mercados laborales... Debiéndose esto a que los individuos se disponen a adquirir mayor escolaridad para competir entre sí por un determinado estatus, lo cual eleva continuamente la demanda educativa, aunque la demanda laboral tienda a permanecer en niveles relativamente estables". Afirma dicha teoría que los nuevos trabajadores están mejor formados que aquellos que van dejando los mismos puestos y que aunque la demanda laboral suele permanecer estable, la demanda educativa 
es cada vez mayor, puesto que hace falta mayor formación para acceder a puestos que antes no la requerían, y todo ello en competencia por un determinado estatus.

Otras dos teorías que se pueden mencionar y sobre las cuales se fundamentan los estudios de egresados son la Teoría de la Fila y la Teoría de la Devaluación de los Certificados. Mientras que la primera establece que "la educación les proporciona a los patrones los elementos para seleccionar a los trabajadores que pueden entrenarse con mayor facilidad, basados en los valores y normas no cognoscitivas que los estudiantes adquieren a medida que avanza en la escuela" (Anuies, 1998). Este enfoque se basa en la teoría del filtro, que afirma que la credencialización académica es la base a través de la cual el empleador está en posibilidad de identificar a las personas que tuvieron mejores desempeños escolares (notas o calificaciones altas) y, por ende, serán estos los individuos más idóneos para ser contratados. Por tanto, a mayor credencialización y desempeño escolar mayores oportunidades de ser contratados. Esta Teoría de la Fila, se fundamenta también en otra teoría, muy relacionada con la anterior: la de la cola. Según esta teoría la escolaridad del individuo juega un papel importante al momento de incorporarse al mercado de trabajo, es decir, los individuos más educados lograrían colocarse primeramente en un empleo obligando a aquellos con menor escolaridad a esperar otras oportunidades de empleos. En concreto, este enfoque afirmaba que a mayor educación, mayor facilidad de empleabilidad. Estas dos teorías sirven de sustento teórico a la Teoría de la Fila que, como hemos dicho, considera que la productividad de un individuo al desempeñar un trabajo no depende de la persona, sino que está condicionada por las características y naturaleza del puesto en cuestión, por lo que el empleador sólo identifica a los individuos que muestren evidencias de ciertas habilidades y destrezas, las mismas que con un entrenamiento eficaz puedan mejorarse y de esta forma aumentar los niveles de productividad en el trabajo. En pocas palabras, este enfoque afirma que a mayor educación mayor facilidad de entrenabilidad en un puesto de trabajo.

Con respecto a la Teoría de la Devaluación de los Certificados, Anuies (1998) señala que "el argumento de la selección se basa en los certificados que les otorgan a los estudiantes a medida que avanzan en la escuela: al existir muchos profesionales demandantes de empleo, el titulo se devalúa, de tal forma que cada vez se requieren más títulos para ocupar un mismo trabajo". En la misma línea, Navarro (1998) sostiene que como los empleadores utilizan como tabla rasa los documentos que los individuos obtienen en la escuela al momento de seleccionar a los trabajadores, es fácil entender que cuando existe una gran demandan de empleos en áreas específicas de formación, los empleadores utilizan la estrategia de devaluar el título o grado académico para detener la demanda de espacios laborales, de esta forma se exige a los candidatos a ocupar un empleo más credencialización académica del que es necesario.

Respecto a la Teoría de la Segmentación, según la cuál no son las características del capital humano las que definen los salarios, sino que será la tecnología utilizada en la empresa la que determine los ingresos de los trabajadores. Lo importante por tanto no es la educación, sino el proceso productivo. Dicha teoría, que surge a finales de los años setenta como crítica a la explicación neoclásica del mercado de trabajo, entiende el mercado de trabajo como un mercado compuesto por un 
conjunto de segmentos distintos, con mecanismos de formación salarial y de asignación diferentes entre sí y con obstáculos a la movilidad entre ellos. La economía ortodoxa, desde su óptica del equilibrio, encontraba dificultades para explicar fenómenos como la persistencia de la pobreza, el desempleo, la discriminación y, sobre todo, las desigualdades salariales entre individuos semejantes. En particular, para la teoría del capital humano las diferencias salariales deberían reflejar diferencias en la productividad (y, en último término, en las cualificaciones); a corto plazo podría haber desigualdades transitorias o fenómenos como el desempleo involuntario, pero a largo plazo la búsqueda de la maximización del beneficio y de la utilidad, en un contexto de información y movilidad perfectas, debería conducir al vaciado del mercado y a la desaparición de las desigualdades. Sin embargo, todo esto chocaba con la realidad y alentaba la búsqueda de explicaciones alternativas. Y entre ellas surge esta teoría de la segmentación del mercado de trabajo (Fernández Huerga, 2010).

La Teoría de la Segmentación se basa, por tanto, en el papel desempeñado por las características propias de cada empleo para la determinación de los salarios y para la productividad atribuida a la fuerza laboral. Esta teoría señala que el mercado laboral está compuesto por tres segmentos con características perfectamente diferenciadas e integrados por grupos no competitivos de trabajadores que comparten algunas características comunes, presentando poca movilidad o transferencia de trabajo entre ellos; dichos segmentos son denominados primario independiente (nivel directivo y de alta gerencia), primario subordinado (nivel ejecutivo y de supervisión) y segmento secundario (nivel operativo). La Teoría de la Segmentación considera, en definitiva, que estos segmentos serán ocupados por los individuos según la división social, sexual y económica a la que pertenezcan en la sociedad (Muñoz, 1985). Esta teoría ha resultado ser una de las más completas para explicar la relación educación-trabajo ya que retoma los fundamentos de las teorías que le antecedieron; refuerza el papel de la educación como proceso de estratificación y reproducción social, afirma que la educación cumple una función distributiva para lograr movilidad social y ocupacional, generando en los individuos la búsqueda de un mayor credencialismo aumentado a la vez, el cisma entre las clases sociales.

Por último, podemos hablar de una de las más recientes teorías que ayudan a interpretar esta relación entre el mundo de la educación y el trabajo. Se trata del Enfoque Alternativo. Esta emergente teoría considera que la influencia de la escolaridad de un individuo no sólo se evidencia en términos de su productividad en el trabajo, sino que de igual manera debe apreciarse en una mejoría en sus capacidades humanas. Este enfoque de capacidades humanas no excluye a los clásicos enfoques económicos como la teoría del capital humano para evaluar el impacto de la educación en el desarrollo, más bien, tiende a complementarlos al estudiar y concentrarse en la relación directa entre la educación y los logros de la libertad humana y, en el papel indirecto que juega en el cambio social y la productividad económica, la adquisición de habilidades, destrezas y conocimientos que se adquieren a través de la escolarización (Flores, 2005).

A pesar de estas teorías y el amplio debate académico existente entre educación y mundo del trabajo, no se puede afirmar que exista un cuerpo de conocimiento sistémico sobre dicha relación. Se entiende que dichos fenómenos no pueden ser 
interpretados solamente a través de la luz de una sola teoría pues de hecho estas relaciones no son directas y unívocas, sino de carácter complejo, en el que intervienen factores tanto escolares como extraescolares. Así pues, para el análisis e interpretación de los resultados de un estudio de seguimiento de egresados resulta conveniente tomar en cuenta los diversos enfoques o teorías a fin de contrastar sus diversos argumentos, categorías y variables y de esta forma encontrar explicaciones más satisfactorias de este objeto de estudio.

De hecho, si nos centramos en los estudios de egresados propiamente dichos, son muchos los estudios realizados por las universidades, que, además, aplican distintas metodologías sin que exista unificación que facilite la comparación de los resultados. Países como Estados Unidos, Canadá y México que cuentan con una gran tradición en este tipo de estudios han llegado a crear un cuerpo unificado de técnicas de recolección de información y han aunado conceptos referentes a las variables de estudio, las metodologías de trabajo...pero, en cualquier caso, estamos hablando de un tipo de investigación que varía mucho según la institución que la realice y es que, el estudio siempre está supeditado a los objetivos o las necesidades que la institución se plantee en un momento determinado.

En cualquier caso, parece que existe cierto consenso entre los estudiosos del tema en cuanto a la aplicación y utilidad que este tipo de investigaciones pueda tener. $Y$ es que "estos estudios constituyen una alternativa para el autoconocimiento y para la planeación de los procesos de mejora y consolidación de las instituciones educativas” (Fresán, 1998).

De ahí que nuestro trabajo trate de evaluar el producto final de la universidad (el egresado) que permita, a su vez, evaluar la titulación impartida para, de esta forma, mejorar de cara a la formación de futuros alumnos y de paso, mejorar la comunicación entre la universidad y los titulados. Todas las consultas efectuadas en torno al estado de la cuestión, parecían aconsejar una mayor cercanía a la sociedad acerca de los parámetros de medida de la calidad universitaria, más allá de aquellos que cuantifican las publicaciones indexadas, los premios, patentes, etc. De ahí que la investigación realizada pretende dar un paso más en esta controvertida vertiente de las valoraciones continuas. Un paso que apuesta por la valoración en su propio contexto, porque si las universidades del sur de Europa tienen que competir con las de Estados Unidos, Japón, etc., la adecuada ponderación estadística parece imponerse, por lo que se apuesta por un posible sistema de medida objetivo, cuantificable y basado en uno de los puntos clave de la responsabilidad universitaria, que no es otro que su capacidad de formar personas capaces de aportar crecimiento y riqueza social.

\section{Metodología}

\subsection{Objetivos}

La naturaleza fundamental de esta investigación ha sido entender la situación de los egresados, sus percepciones con respecto a su formación y su situación laboral para, de esta forma, llegar a conocer el recorrido laboral y académico de los 
titulados. No es suficiente con obtener información sobre el número de egresados que está trabajando, sino que hay que valorar cuestiones como la estabilidad laboral, la remuneración obtenida por el desempeño laboral o el grado de satisfacción que los licenciados tienen en sus puestos de trabajo y que deberían ser también tenidos en cuenta. La pregunta de investigación, que fundamenta este estudio es: ¿qué ha sido de los egresados de Publicidad y Relaciones Públicas de la Universidad de Cádiz?

Para dar respuesta a esta pregunta, se plantea como OBJETIVO GENERAL de este trabajo conocer la opinión y la situación profesional del egresado de la Licenciatura en Publicidad y Relaciones Públicas de la Universidad de Cádiz a fin de contribuir a la mejora continua de estos estudios y al desarrollo de los mismos.

Entre los OBJETIVOS ESPECÍFICOS, podríamos destacar:

- $\quad$ Conocer las fortalezas y debilidades en la formación del egresado

- Evaluar la relación existente entre los programas formativos desarrollados y el empleo de los titulados

- Caracterizar la trayectoria laboral así como el proceso de inserción laboral de los egresados

- C Conocer las características del "primer empleo" de los egresados

- Conocer las características del empleo actual y su valoración

- Conocer el grado de satisfacción del egresado respecto a la calidad de la formación que ha recibido durante su carrera y la adecuación a su experiencia laboral

- $\quad$ Evaluar la adecuación del trabajo a los estudios realizados

\subsection{Diseño de la investigación}

El propósito fundamental de este "estudio de egresados" es hacer un seguimiento de los licenciados en Publicidad y Relaciones Públicas durante los cursos 2005-2007, 2006-2008 y 2007-2009. Nuestro interés se centra en conocer qué ha sucedido con la población compuesta por estudiantes de tres promociones y, para ello, vamos a tener en cuenta y vamos a estudiar variables relacionadas con la inserción laboral y la experiencia en el mundo laboral y sus implicaciones académico-formativas.

Para la planificación y el posterior desarrollo del estudio, se siguieron los siguientes pasos:

En primer lugar, se plantearon los objetivos que se pretendían alcanzar y se decidió quiénes iban a ser los individuos objeto del estudio. 
En segundo lugar, se procedió a realizar una revisión de estudios sobre inserción laboral realizados por otras universidades. Tras esta recopilación de trabajos y la consiguiente revisión de los mismos, se consideró el método de encuesta como el principal instrumento metodológico, puesto que en los estudios de seguimiento a egresados es la herramienta más utilizada. La utilización de la encuesta, implementada a través del cuestionario, es una herramienta muy útil para indagar en las percepciones del egresado en cuanto a la formación que han recibido y también para entender el recorrido laboral y académico que los egresados han desarrollado toda vez que han conseguido su título universitario. De ahí que para obtener la información de nuestra muestra hayamos optado por este procedimiento.

Una vez que se decidió que para realizar el estudio, el método empleado sería el de encuesta, hubo que considerar que dentro del método de encuestas existen diversos sistemas para aplicar las mismas, por lo que había que seleccionar el más adecuado para cumplir con los objetivos de la investigación. Uno de estos sistemas consiste en el envío del formulario por correo postal, que según Cabrera es con el que mejores resultados se obtienen. El problema que suscita este sistema es que es necesario tener las direcciones de residencia de todos los componentes de la muestra y esta información, en muchos casos, es difícil de conseguir. Además, el bajo índice de respuesta es otra gran desventaja de este sistema.

Una alternativa es la utilización de la entrevista telefónica, pero este método es caro, y aunque se consigue un mayor índice de respuesta que con el envío postal, también es complicado conseguir los números de teléfono de la población objeto de estudio.

Finalmente y aprovechando las nuevas tecnologías, contamos con dos nuevas modalidades: el uso de la página web o el correo electrónico. Con estas herramientas, podemos obtener la información con un bajo costo, incrementamos el índice de respuesta además de poder tabular los datos de forma inmediata. Es importante motivar al egresado en la fase de recolección de la información, para que entre en la encuesta y conteste al cuestionario pero, una vez rellena el mismo, el investigador recibe la información para su procesado y análisis de manera inmediata.

Teniendo en cuenta las características de este trabajo, el presupuesto con el que se contaba para desarrollarlo y la información con la que se contaba sobre los egresados, se optó por la página web como método de recogida de la información. Para ello se utilizó la herramienta que proporciona la página web de encuestas online (www.encuestafácil.com), página web que facilita el desarrollo de las encuestas a través de la elaboración de los cuestionarios para implementar las mismas, recogida de información en línea, el procesamiento de los datos y la generación de tablas estadísticas básicas, para posteriormente ser tratados estadísticamente con los programas Microsoft Excel 2007 y el software estadístico SPSS.

Una vez decidido el método de encuesta como el ideal para desarrollar esta investigación y el sistema para la recogida de datos, se procedió a la elaboración del cuestionario que iba a aplicarse en la recogida de datos. 
El trabajo comienza con la fase de diseño. En primer término, se realizó un diseño inicial del cuestionario, diseño que se constituyó como la primera versión del mismo.

Esta primera versión es analizada y, además, se realiza una sencilla simulación con ex alumnos. Tras ser considerado excesivamente largo, es revisado para su mejora. Acorde con estas valoraciones, dicho cuestionario inicial se modifica para, una vez concluida la revisión, iniciar los primeros ensayos con el cuestionario definitivo terminando, de esta manera, la fase de validación.

Dicho cuestionario definitivo quedó estructurado de acuerdo con el siguiente esquema:

- $\quad$ información personal

- $\quad$ otros estudios comenzados por los alumnos

- $\quad$ primer empleo

- $\quad$ situación actual

- $\quad$ organización en la que trabaja el egresado

- $\quad$ evaluación de la carrera

Una vez validado el cuestionario el siguiente paso consistió en la selección de la muestra. Dicha muestra se extrajo del universo conformado por los egresados de la carrera Licenciatura en Publicidad y Relaciones Públicas, en los ciclos de egreso 2005-2007, 2006-2008 y 2007-2009, y que en total suman 180 alumnos, distribuyéndose dichos alumnos según la promoción de la siguiente forma:

Cuadro 1. Tamaño de la población objeto de estudio

\begin{tabular}{|l|l|}
\hline Número de egresados por promoción & 48 \\
\hline $2005-2007$ & 52 \\
\hline $2006-2008$ & 80 \\
\hline $2007-2009$ & 180 \\
\hline TOTAL & \\
\hline
\end{tabular}

Fuente: Elaboración propia

De este universo de 180 alumnos, se consiguió el correo electrónico de 161 de ellos, fijándose por tanto la muestra definitiva, conjunto final de personas encuestadas, en esos 161 egresados, lo que supone un 90\% de la población (180 personas). De dicha muestra inicial con la que se intentó contactar 111 personas accedieron a la web y respondieron el cuestionario 
lo que implica una tasa de respuesta del $68 \%$ de la población. A título orientativo, asumiendo que se cumplen los requisitos del muestreo aleatorio simple, para este plan de muestreo se ha considerado un error muestral del $10 \%$, un nivel de confianza del $90 \%$ y un valor de precisión B de 0,05

Por último, se pasó a la fase de aplicación. Para la puesta en marcha del cuestionario de egresados se pusieron en práctica las siguientes actividades:

Se contactó con cada participante vía correo electrónico para hacerle partícipe del estudio y solicitar su colaboración. Con dicho correo se daba a conocer el objetivo principal de la investigación, además de proporcionarle el enlace a la web donde estaba hospedada la encuesta para que fuese contestada. Además, se registraba el acuse de recibo de cada participante, de cara al control. Transcurrida la primera semana, se volvió a enviar otro correo electrónico para intentar aumentar el índice de respuesta.

La encuesta estuvo abierta durante 90 días, para que los participantes pudieran contestarla. Una vez transcurrido dicho plazo, se cerró y se procedió a la recogida y tratamiento de la información, para, finalmente, realizar el análisis de los resultados más relevantes del estudio. Como se ha dicho, la tabulación de datos, el cálculo de indicadores y el análisis estadístico se realizaron utilizando los paquetes SPSS y Excel.

\section{Análisis de resultados}

\subsection{Perfiles sociodemográficos}

Como primera toma de contacto, se muestran los resultados generales obtenidos. En el primero de los gráficos (Gráfico 1) se hace referencia a la respuesta obtenida según el sexo, observándose un mayor porcentaje de mujeres que de hombres. El 66\% de los egresados son mujeres. Este dato está en línea con los datos de distribución por sexos de la población y, además, evidencia una manifiesta "feminización” del alumnado de las universidades. Según el Ministerio de Educación en su estudio sobre el Sistema Universitario Español sobre el curso 2011-2012 el 54,1\% del total de estudiantes universitarios son mujeres, su presencia es mayoritaria en todos los niveles de formación universitaria. La proporción de mujeres se incrementa entre los titulados universitarios hasta situarse en el 59,7\% de media. En la rama concreta de las Ciencias Sociales, está cifra es del $61,3 \%^{1}$

1 Fuente: Ministerio de Educación, Cultura y Deporte. Datos y Cifras del Sistema Universitario Español. Curso 2011-2012 
Cuadro 2. Sexo de los egresados

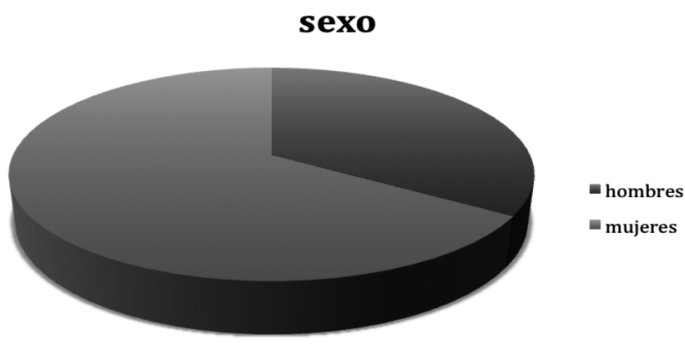

Fuente: elaboración propia

La edad media de los encuestados resulta elevada (25,6 años). Es un dato lógico, si tenemos en cuenta que se trata de una carrera de segundo ciclo, que complementa a otros estudios universitarios cursados por los encuestados. La presencia de estudiantes mayores de 30 años cada vez es más intensa entre los estudiantes universitarios. Así, mientras que en el curso 2000-01 el 9,5\% de los estudiantes de primer y segundo ciclo tenían más de 30 años, en el curso 2010-2011 su presencia se duplica alcanzando el $18,2 \%$

Se quería conocer, además si los estudios eran su actividad principal o, al tratarse de una carrera de segundo ciclo, ya formaban parte del mundo laboral. Y, además, se quería saber si los egresados tenían experiencia laboral previa, ya fuese relacionada con los estudios o no.

Para el 59\% de los encuestados, los estudios eran su actividad principal, pero, antes de acceder a la carrera de Publicidad, el $75 \%$ ya contaba con experiencia laboral, de los cuales, el 17\% tenía experiencia laboral relacionada con la carrera. En cualquier caso, el porcentaje de alumnos que se dedican en exclusiva a cursar la carrera es elevado, teniendo en cuenta que se trata de personas que ya están en edad de trabajar. Entre la población de encuestados que tiene esa experiencia laboral, se observan diferencias significativas entre hombres y mujeres, siendo las personas de sexo varón las que con mayor frecuencia tienen empleo mientras cursaban estos estudios. 
Cuadro 3. Experiencia laboral previa no relacionada con los estudios

\section{Experiencia laboral previa no relacionada con los estudios}

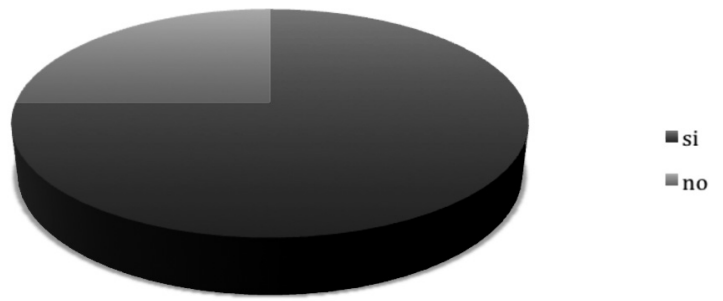

Fuente: elaboración propia

Cuadro 4. Experiencia laboral previa relacionada con los estudios

\section{Experiencia laboral previa relacionada con los estudios}

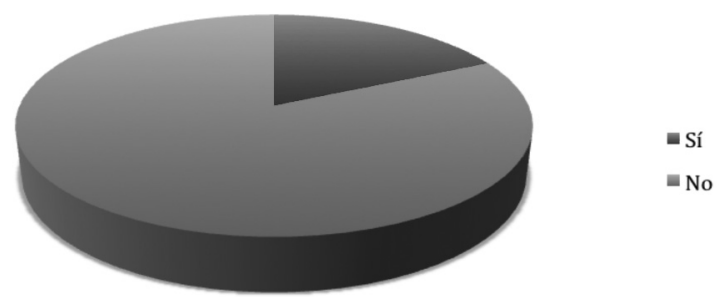

Fuente: elaboración propia

Cuadro 5. Situación en el último año de carrera

\section{Situación en el último año de carrera}

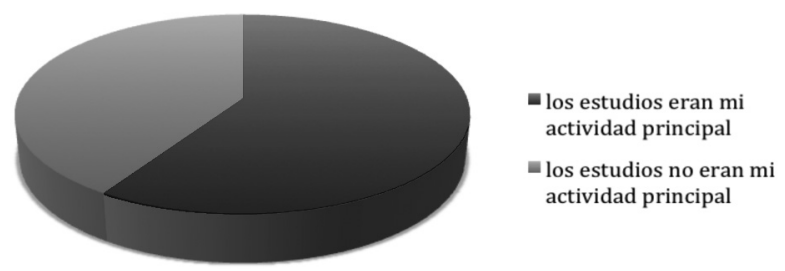

Fuente: elaboración propia 
Cuadro 6. Situación en el último año de carrera por sexos

\section{Situación en el último año de carrera por sexos}

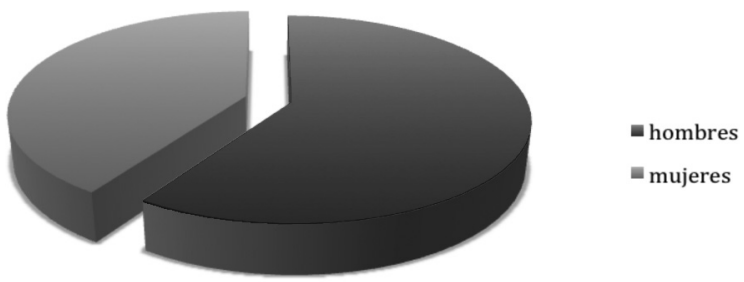

Fuente: elaboración propia

- Otros estudios comenzados por los alumnos

También queríamos saber si además de cursar la carrera de Publicidad, los egresados habían seguido formándose, de cara a mejorar sus expectativas laborales y completar aquellas deficiencias que, de alguna manera, hubiesen podido encontrar a lo largo de su formación universitaria.

Cuadro 7. Otros estudios comenzados por los egresados tras la graduación

Otros estudios comenzados tras graduarse

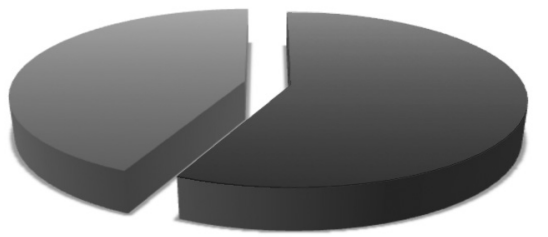

Fuente: elaboración propia

Y, efectivamente, el 57\% ha empezado otros estudios orientados a la obtención de algún otro título. El 29\% de dichos egresados ha comenzado algún estudio de licenciatura, ingeniería o arquitectura, mientras que el $31 \%$ lo ha hecho de diplomatura. Por tanto, el $60 \%$ de los encuestados que ha seguido estudiando se ha decantado por una carrera universitaria. El 20\% restante ha optado por cursar algún master, mientras que un 12\% comenzó estudios de idiomas. 
Cuadro 8. Tipo de estudios iniciados por los egresados

Tipo de estudios

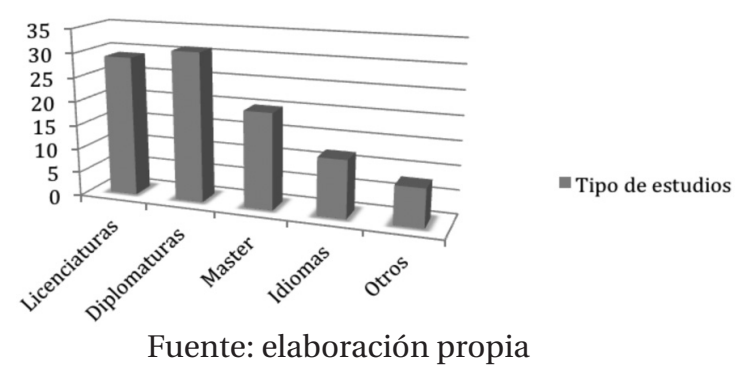

La distribución por sexos muestra que las mujeres son más proclives a seguir formándose, siendo mayor el porcentaje de mujeres (59\%) que el de los hombres.

Cuadro 9. Otros estudios comenzados por los egresados por sexos

\section{Otros estudios comenzados tras} graduarse por sexos

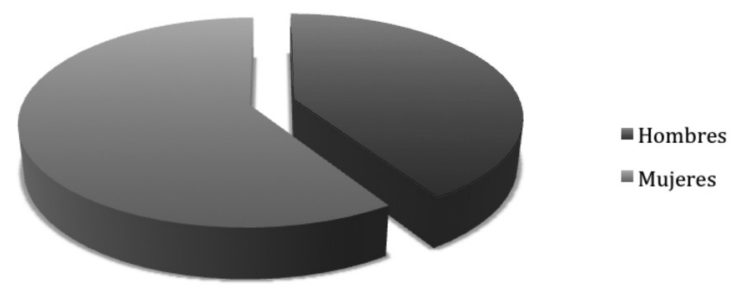

Fuente: elaboración propia

Cuadro 10. ¿Has finalizado los estudios?

Finalización de los estudios

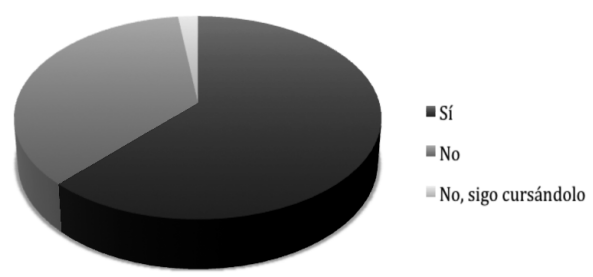

Fuente: elaboración propia 
El 62\% del total ha finalizado dichos estudios, sólo el 2\% los abandonó y el resto, un 36\%, sigue cursando sus estudios.

Por sexos, las mujeres realizan con mayor frecuencia estudios de masters e idiomas, mientras que los hombres se decantan mayoritariamente por comenzar otro tipo de estudios universitarios.

Cuadro 11. Tipo de estudios iniciados por los egresados por sexos

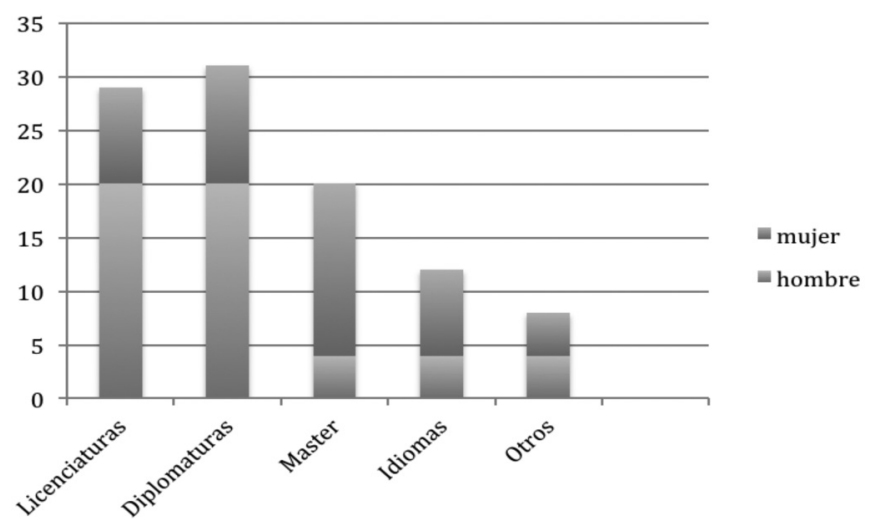

Fuente: elaboración propia

\section{- Primer empleo}

El 82\% de los encuestados ha trabajado desde que se graduó. De este porcentaje, el 50\% empezó a trabajar tras la obtención del título y el 32\% restante siguió trabajando en el empleo que ya tenían. Es interesante que sólo el 18\% de los egresados no haya trabajado desde que se graduó, hasta la fecha de la encuesta.

Cuadro 12. ¿Has trabajado al finalizar los estudios?

\section{Trabajo al finalizar los estudios}

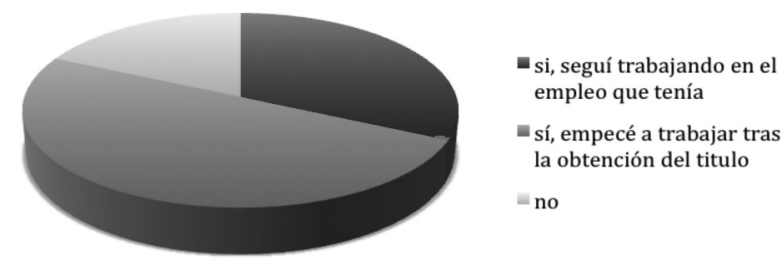

Fuente: elaboración propia 
Encuestados sobre su primer empleo, pusimos especial interés en conocer cuál era el grado de relación entre este primer trabajo y los estudios de Publicidad y Relaciones Públicas. Como dato relevante, el $41 \%$ de los encuestados trabajó en algo que guardaba mediana coincidencia con los estudios de Publicidad y Relaciones Públicas, siendo total la coincidencia en el $17 \%$ de las encuestas, por lo que el $58 \%$ de los primeros empleos de los encuestados ha tenido que ver con la carrera de Publicidad y Relaciones Públicas.

Sólo el $24 \%$ tuvo nula coincidencia y un 17\% considera que la coincidencia entre su primer empleo y los estudios cursados fue baja.

Cuadro 13. Grado de coincidencia estudios-trabajo

\section{Grado de coincidencia estudios- trabajo}

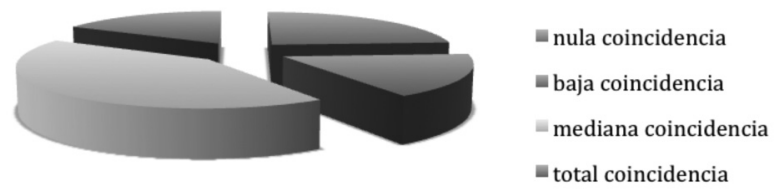

Fuente: elaboración propia

En cuanto a las condiciones de este primer empleo, el 51\% tenía contrato de duración limitada, y sólo el 19\% tuvo un contrato indefinido. El 30\% restante tuvo otro tipo de contrato, como becario, en prácticas...

Cuadro 14. Tipo de contrato primer empleo

\section{Tipo de contrato}

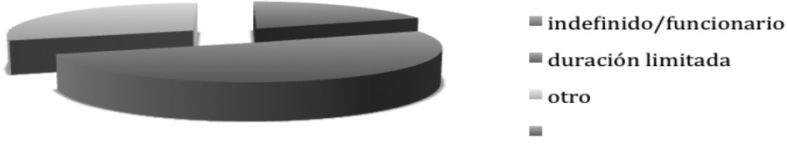

Fuente: elaboración propia

Con respecto al salario, una cifra muy alta, el 76\% percibía en su primer empleo menos de mil euros. De hecho, el 38\% percibía menos de 500 euros en su primer trabajo. Un $20 \%$ de los encuestados cobraba entre mil y 1500 euros y un dato curioso: 
sólo un 5\% de los encuestados percibía mas de 1500 euros. Como medida de comparación se podría utilizar el estudio de la Facultad de Ciencias de la Comunicación de Santiago de Compostela del año 2004, extraído del Libro Blanco, Títulos de Grado en Comunicación, en el que se comprueba como un 57\% de los licenciados tenía un salario inferior a 900 euros, un $27 \%$ cobraba entre 900 y 1.200 euros y tan sólo el 16\% obtenía unos ingresos mensuales superiores a los 1.200 euros.

Cuadro 15. Salario primer empleo

\section{Salario}

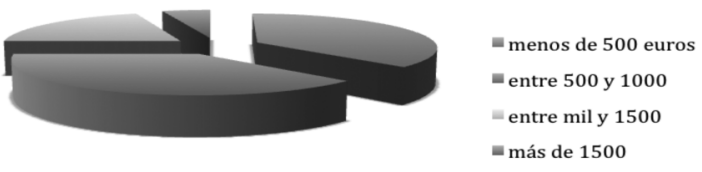

Fuente: elaboración propia

En lo respectivo al salario mensual, se han observado diferencias entre mujeres y hombres: por regla general ellas cobraban menos que ellos. Es destacable el hecho de que las diferencias entre el grupo de los que cobraba más de 1.500 euros se decanta a favor de las mujeres.

Cuadro 16. Salario primer empleo en función del sexo

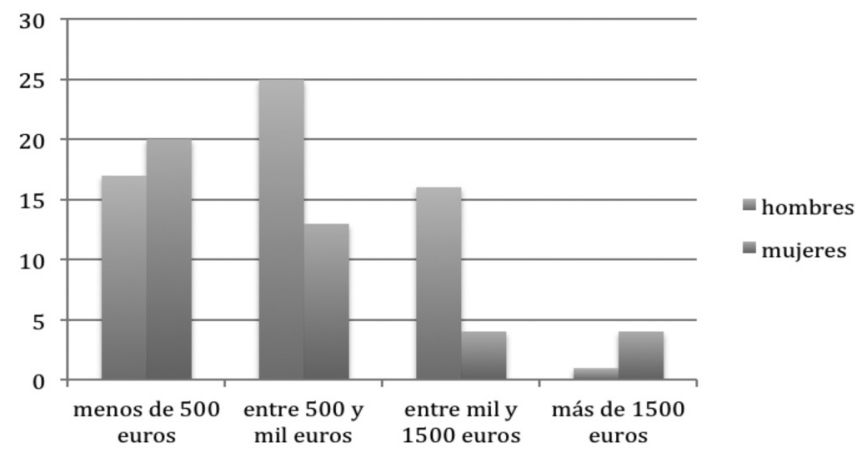

Fuente: elaboración propia

El 54\% de los encuestados que encontró trabajo tardó menos de 6 meses en hacerlo, siendo un 29\% el porcentaje de encuestados que lo encontró en menos de 3 meses. Un $6 \%$ tardó entre 6 meses y un año y un 10\% tardó mas de un año en hacerlo. Estos datos de incorporación al mercado laboral son peores que los que muestra un estudio de la Universidad Complutense de Madrid llevado a cabo en 2004 y según el cual, el 72,7\% de los egresados en Publicidad y Relaciones Pú- 
blicas tardaron menos de 3 meses en insertarse al mercado laboral después de finalizar sus estudios oficiales. De hecho, el 29,5\% se colocó nada más finalizar la carrera.

Cuadro 17. Tiempo transcurrido hasta que el egresado encontró el primer empleo

\section{Tiempo hasta que encontraste ese trabajo}

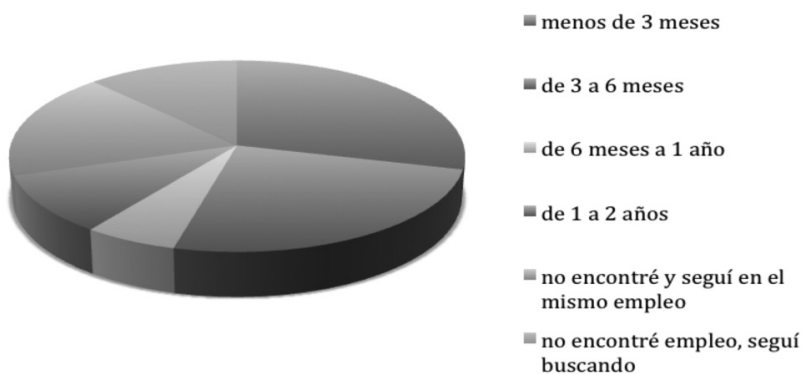

Fuente: elaboración propia

Por sexos, entre el grupo de mujeres parece que hay más problemas a la hora de encontrar empleo, de modo que se observan porcentajes inferiores en la respuesta "menos de tres meses".

Cuadro 18. Tiempo transcurrido hasta que el egresado encontró el primer empleo en función del sexo

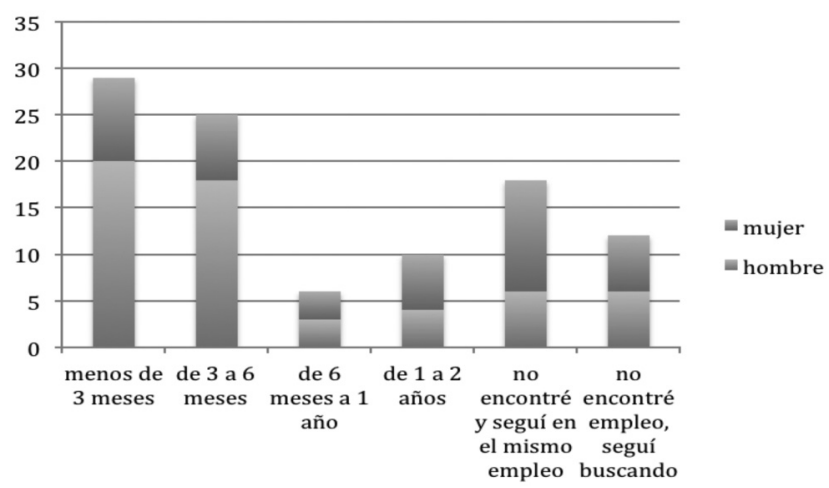

Fuente: elaboración propia

Un estudio llevado a cabo por la Escuela Universitaria de Estadística de la Universidad Complutense en 2003 y citado por Cinco Días señala que el 80\% de los titulados en Periodismo se coloca en el plazo de un mes. La Universidad SEK de Se- 
govia realizó una encuesta a sus licenciados y el resultado para la respuestas "Tres meses o menos" hasta encontrar trabajo es del $75,6 \%$. Hemos de señalar que estos estudios están realizados con estudiantes de Periodismo, y que además son previos a la crisis, lo que puede explicar las diferencias entre unos y otros a la hora de encontrar ese primer empleo tras titularse. A pesar de eso, queríamos comparar los mismos para intentar ver la evolución y las consecuencias de la crisis en la incorporación al mercado laboral de los egresados de una carrera de comunicación.

Preguntados sobre las dificultades y demora a la hora de encontrar ese trabajo la mayoría, el 46\%, considera la escasa experiencia laboral como el principal problema. El 29\% lo achaca a la crisis económica, y sólo el 7\% considera que el problema fue el poco conocimiento de la carrera que tienen los empleadores.

Cuadro 19. Dificultad para encontrar el primer empleo

\section{Dificultad para encontrar trabajo}

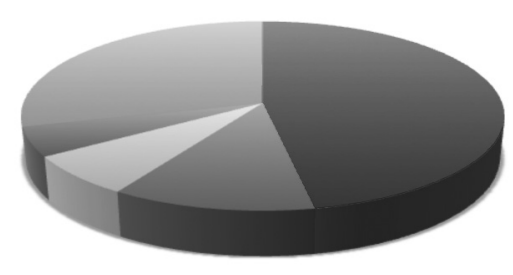

Escasa experiencia laboral

Tenía ofertas de trabajo poco atractivas

- La carrera es poco conocida

- Su situación personal se lo dificultó

Otros

Fuente: elaboración propia

Por último, se preguntó a los egresados si actualmente continuaban en ese trabajo, y el 56\% contestó de manera afirmativa.

Cuadro 20. ¿Continúas en este primer trabajo?

¿Continuas en este primer trabajo?

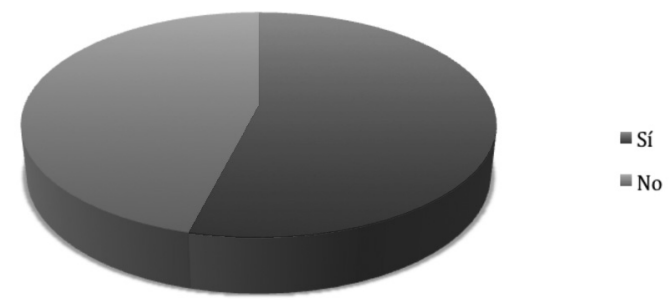

Fuente: elaboración propia 


\subsection{Trayectorias laborales}

- Situación actual

Esta parte de la encuesta hace referencia a la situación laboral actual de los egresados en Publicidad y Relaciones Públicas.

Y lo primero a comentar es que el $82 \%$ de los encuestados está trabajando en el momento de realización de la encuesta. Sólo el 18\% se encuentra sin trabajo. Este dato coincide con el dato extraído del citado estudio del Ministerio de Educación, Cultura y Deporte, según el cual aunque la tasa de desempleo ha aumentado para toda la población, el impacto ha sido desigual en función de la edad y del nivel de formación alcanzado. Así, mientras que el 25,2\% de los jóvenes entre 25 y 29 años están en desempleo, entre los universitarios ese porcentaje se sitúa en el 18\%.

Cuadro 21. Trabajo actual

\section{¿Actualmente tienes trabajo?}

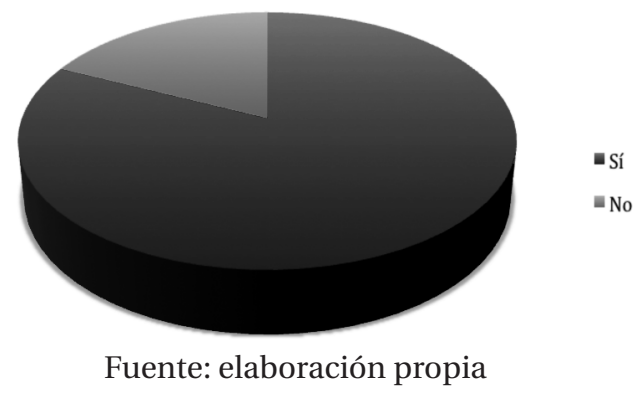

Si comparamos esta información con un estudio llevado a cabo por la Facultad de Ciencias de la Comunicación de la UAB en el año 2000, cuyas conclusiones más relevantes se encuentran en el mencionado Libro Blanco de los Estudios de Grado en Comunicación, podemos comprobar como los resultados son prácticamente idénticos. En dicho estudio, el 82,4\% de los licenciados declaró estar trabajando, frente a un 17,6\% que no. Otro estudio más actual, el anteriormente citado de la Universidad Complutense de Madrid de 2004, ofrece cifras similares, situando el porcentaje de titulados en Publicidad y RRPP que se encontraba en situación laboral activa en el $81,1 \%$, frente al 9,5\% de desempleados y un 9,5\% que estudia.

En cuanto al tipo de contrato, el 28\% cuenta con un contrato indefinido. El 33\% tiene contrato de duración limitada y el $39 \%$ restante tiene otro tipo de contrato. Aunque se trata de un estudio de 1998, en la encuesta CHEERS, el 51,9\% de los licenciados en Comunicación tienen un contrato indefinido. En un estudio más reciente, el llevado a cabo en la Facultad de Ciencias de la Comunicación de Santiago de Compostela en el año 2004, el 43,6\% de los contratos son temporales, el $31,4 \%$ son fijos y el 2,7\% de los licenciados cuenta con otro tipo de contrato. En comparación, los datos presentados por 
el estudio de la Universidad Complutense para sus egresados en Publicidad y Relaciones Públicas son mucho más positivos: nueve de cada diez titulados tiene un empleo a tiempo completo.

Cuadro 22. Tipo de contrato del trabajo actual

\section{Tipo de contrato}

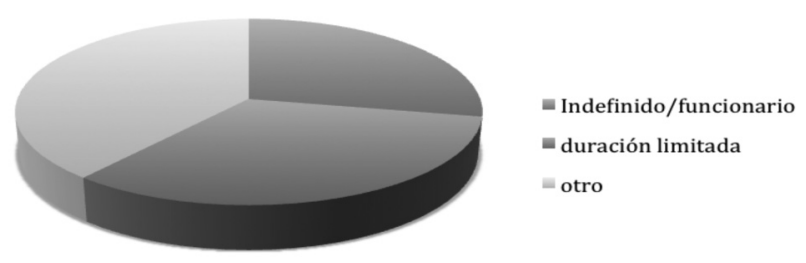

Fuente: elaboración propia

Por sexos, encontramos notables diferencias entre hombres y mujeres en el caso de los contratos indefinidos, siendo los hombres los que obtienen mayoritariamente este tipo de contratos.

Cuadro 23. Tipo de contrato del trabajo actual por sexos

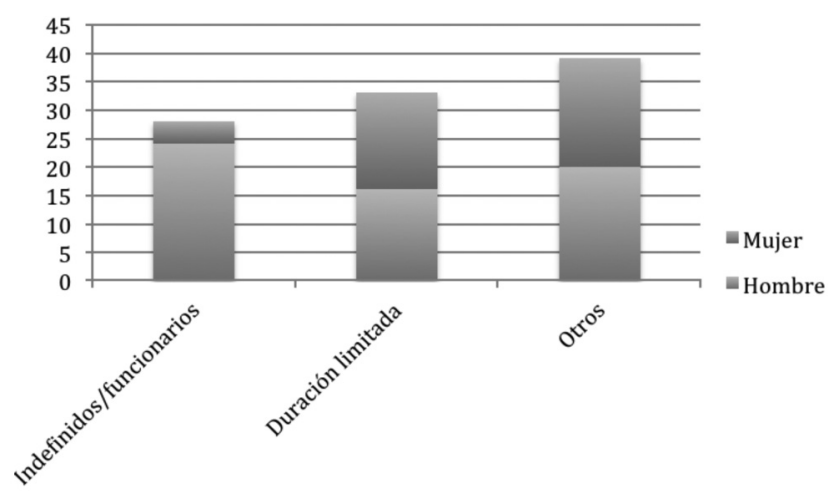

Fuente: elaboración propia

De este $82 \%$ de egresados con trabajo en la actualidad, el $92 \%$ está satisfecho con dicho empleo. De hecho, el $46 \%$ está bastante satisfecho y el $11 \%$ muy satisfecho.

También debe destacarse que el $43 \%$ de los encuestados considera que su trabajo está muy relacionado con su título profesional, y un $41 \%$ considera mediana está relación. 


\section{Cuadro 24. Relación estudios-trabajo actual}

\section{Relación estudios-trabajo}

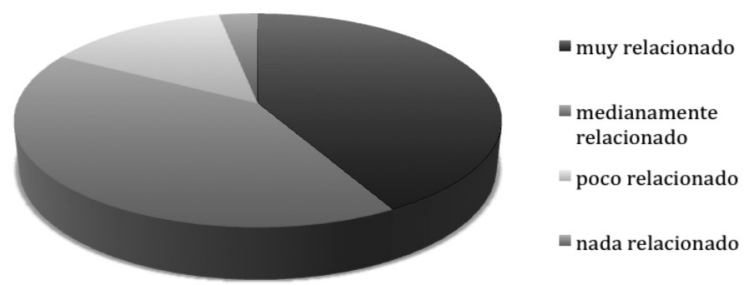

Fuente: elaboración propia

Sólo un 3\% no trabaja en nada relacionado con su título profesional, mientras que un 14\% trabaja en algo cuya relación con el título de Publicidad y Relaciones Públicas es escasa.

Como dato comparativo, señalar en cuanto a la valoración del grado de adecuación de la carrera a las necesidades del mundo laboral, que los licenciados en la UAB en el año 2000, consideran en un 57,9\% que su trabajo está relacionado con la licenciatura estudiada, mientras que el resto considera que no (estudio de 2004). El estudio de la Universidad de Santiago de Compostela arroja datos más favorables: los licenciados puntuaron con un 7,1 el grado de adecuación de la carrera con respecto a las necesidades laborales. El estudio de la Universidad Complutense de Madrid cifra este porcentaje de egresados que están empleados en ocupaciones relacionadas con la formación recibida en más del $60 \%$. De hecho, cuando se les pide que valoren la relación entre el trabajo que realizan en la actualidad y su formación, utilizando una escala de 0 a 10, los egresados en Publicidad la valoran con un 6,9 de media.

En este sentido, es interesante la opinión del profesor Díaz Nosty para quien "los planteamientos de las facultades de comunicación son aún desfasados y escasamente dinámicos, puesto que las necesidades del mercado avanzan mucho más rápidamente que los planes de estudios” (Libro Blanco, Títulos de Grado en Comunicación, 2005: 183).

Además de desempeñar un trabajo, el 64\% ha realizado alguna actividad formativa relacionada con su tarea profesional en los últimos doce meses. 
Cuadro 25. Realización de actividades formativas

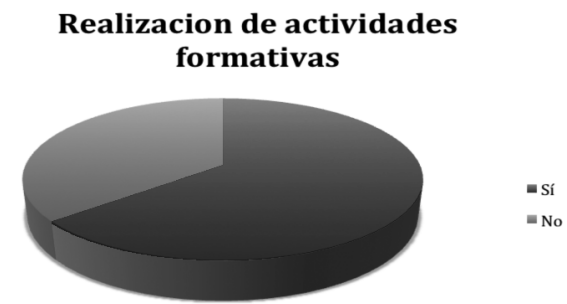

Fuente: elaboración propia

Por sexos el dato es llamativo, pues es mayoría el porcentaje de mujeres que ha desarrollado alguna actividad formativa respecto al de los hombres.

Cuadro 26. Realización de actividades formativas

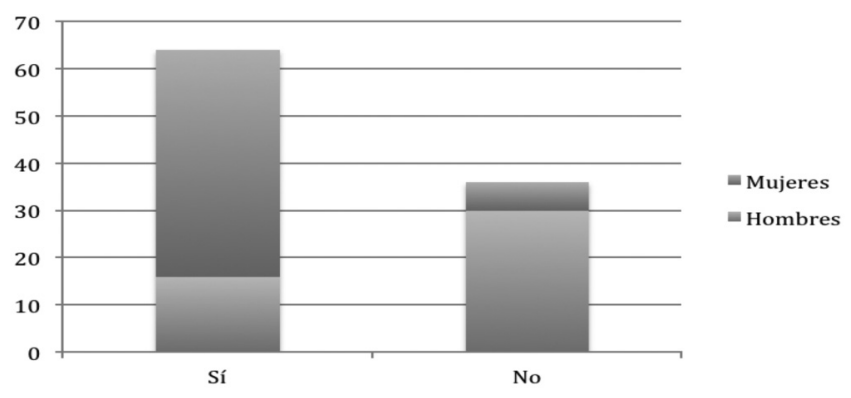

Fuente: elaboración propia

En cuanto a las expectativas laborales para el futuro, el 38\% aspira a un ascenso o promoción en el mismo lugar de trabajo, y un $14 \%$ busca la estabilidad en su puesto.

El porcentaje de encuestados que aspira a una promoción o cambio a un mejor puesto es elevado: 41\%. Mientras que el $8 \%$ vive una situación de incertidumbre y de riesgo de despido. 
Cuado 27. Expectativas laborales de los egresados

\section{Expectativas laborales}

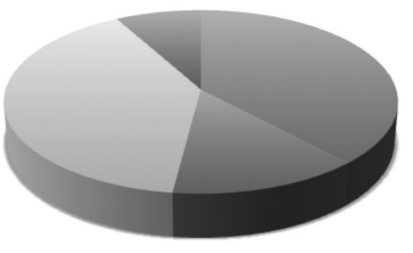

= Ascenso o promoción

estabilidad

cambio a un lugar mejor de trabajo

- incertidumbre, riesgo de

despido

Fuente: elaboración propia

- Organización en la que trabaja el egresado

También hemos querido, en esta investigación, conocer algo del entorno en el que trabajan nuestros egresados por lo que les hemos preguntado sobre su organización.

Así, el 78\% trabaja en una empresa del sector privado. Sólo el 19\% de los encuestados trabaja en la empresa/administración pública. Hay un 3\% de nuestros egresados que trabaja para empresas privadas sin ánimo de lucro.

Cuadro 28. Sector de la organización en la que trabaja el egresado

Sector de la organización

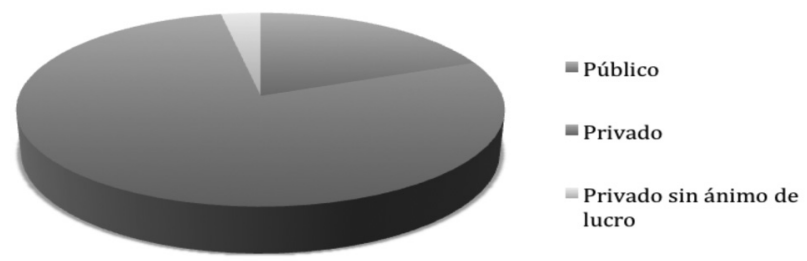

Fuente: elaboración propia

Destaca un aspecto en la diferenciación por sexos, siendo mayor el porcentaje de mujeres que trabajan en el sector público. 
Cuadro 29. Sector de la organización en la que trabaja el egresado por sexos

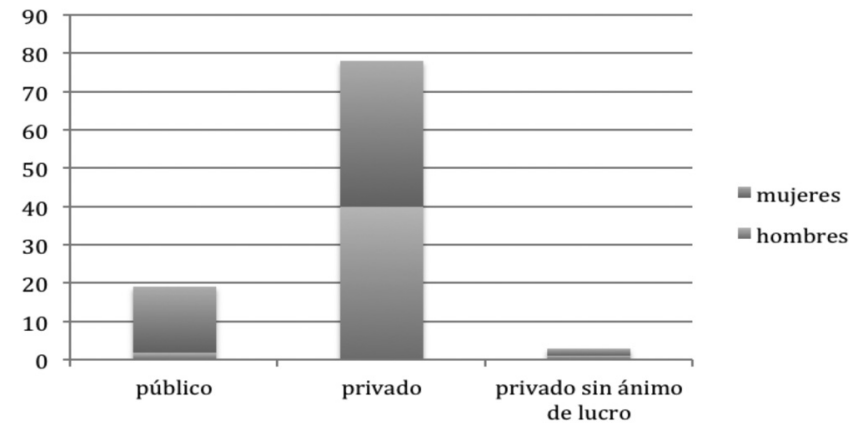

Fuente: elaboración propia

De estas empresas, el 31\% desarrolla su actividad en el ámbito internacional. El 25\% de las empresas en las que trabajan los egresados es de ámbito sólo local, mientras que el 22\% es regional. El 22\% restante desempeña su trabajo en empresas de ámbito nacional.

Cuadro 30. Ámbito geográfico de la empresa en que desempeña su trabajo

\section{ámbito geográfico de la empresa}

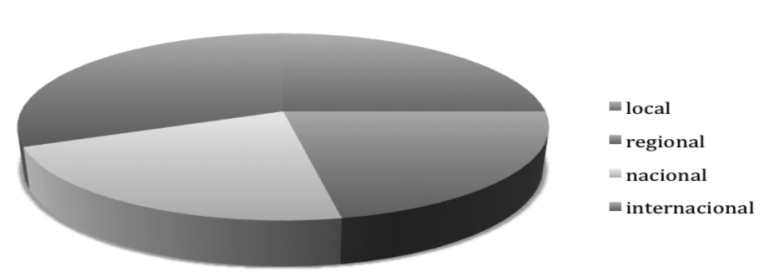

Fuente: elaboración propia

Aquí también se detectan importantes diferencias por sexos. Las mujeres desempeñan su trabajo sobre todo en empresas de ámbito nacional e internacional, mientras que el porcentaje de hombres es mayor en los ámbitos local y regional. 
Cuadro 31. Ámbito geográfico de la empresa en que desempeña su trabajo por sexos

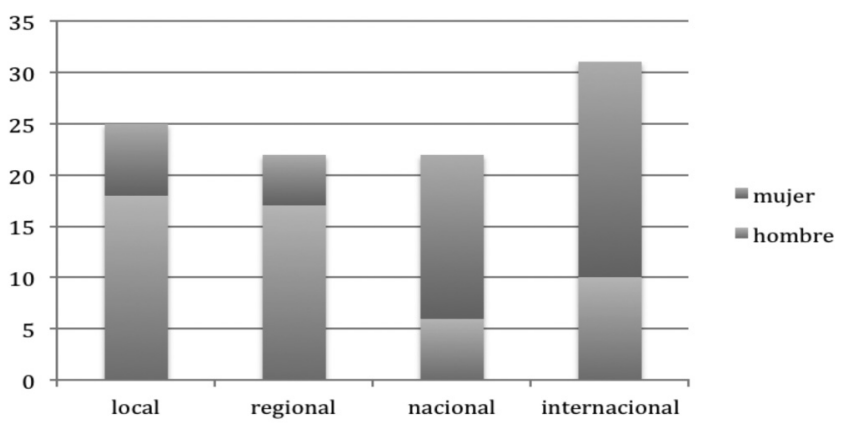

Fuente: elaboración propia

Con respecto al número de trabajadores la mayoría, un 59\%, trabaja en empresas con menos de 50 trabajadores. De hecho, el 31\% trabaja en empresas con entre 1-9 empleados. Un 23\% de los encuestados trabaja en organizaciones con entre 50249 trabajadores y sólo un 18\% lo hace para empresas que cuentan con más de 250 trabajadores.

Cuadro 32. Tamaño de la empresa

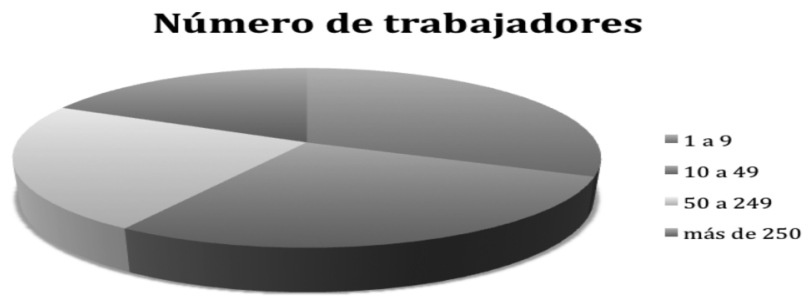

Fuente: elaboración propia

\subsection{Evaluación de la titulación}

Una vez que conocemos la situación laboral de los egresados en Publicidad y Relaciones Públicas de la Universidad de Cádiz, queríamos interrogarlos sobre sus puntos de vista acerca de la formación que habían recibido en la Universidad. De esta forma, el $22 \%$ considera que la formación recibida en la Universidad fue alta, mientras que el 59\% piensa que la formación recibida fue suficiente. Sólo el 15\% piensa que esta formación fue baja. 
Cuadro 33. Formación recibida

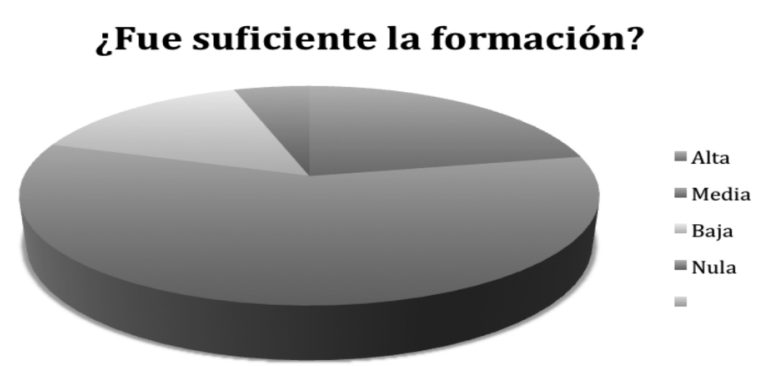

Fuente: elaboración propia

El estudio llevado a cabo por la Facultad de Ciencias de la Comunicación de la Universidad Autónoma de Barcelona muestra como, en el caso de los graduados en Publicidad y Relaciones Públicas, más de la mitad de los encuestados evaluó como satisfactoria su carrera $(51,4 \%)$. El $41,5 \%$ se declaró ni mucho ni poco satisfechos con sus estudios y el resto se posicionó en los extremos: $2,4 \%$ muy satisfactorio, $2,4 \%$ poco satisfactorio y $2,4 \%$ insatisfactorio.

Volviendo a nuestra investigación el 59\% de los egresados, si pudiera volver atrás, volvería a elegir la misma carrera en la misma universidad. Sólo el 12\% estudiaría la misma carrera en otra universidad, y un 17\% se decantaría por otra carrera.

Cuadro 34. Valoración de la carrera

\section{¿Volverías a elegir esta carrera?}

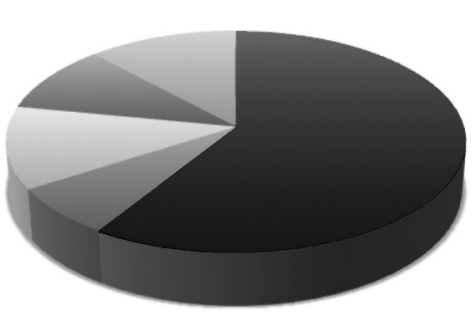

Fuente: elaboración propia
- la misma carrera en la misma universidad

una carrera distinta en la misma universidad

la misma carrera en otra universidad

- una carrera distinta en otra universidad

no estudiaria una carrera universitaria

\section{Discusión de los resultados}

La importancia de la inserción laboral de los universitarios y la preocupación que suscita entre familias, agentes sociales, gestores universitarios y Administraciones Públicas hace que las Universidades se interesen por el seguimiento de 
sus titulados. Esta preocupación por saber qué ocurre tras la graduación y cómo afrontan los egresados la incorporación al trabajo ha supuesto que proliferen los estudios sobre inserción laboral, ya sea por titulaciones ya sea con carácter general.

La investigación que nos ocupa se refiere a un estudio de egresados. Tenemos que distinguir entre un estudio de egresados propiamente dicho, en el que la investigación se realiza en un momento concreto en el tiempo y los estudios de seguimiento de egresados, en los que el seguimiento al egresado se realiza de manera longitudinal, consiguiendo información del mismo en al menos dos momentos posteriores al egreso. Si bien los estudios que se centran en seguir a los egresados son un valioso instrumento para entender al egresado en su inserción al mercado laboral debemos entender que los estudios de egresados tienen una serie de limitaciones: hay pocas posibilidades de compararlo con otros estudios, por las diferentes metodologías y marcos muestrales usados, además es difícil comprobar la información conseguida, sobre todo en temas como las tasas de ocupación o desempleo, por lo que se dificulta comprobar la validez del mismo. Así, la definición de la población objetivo, los años o promociones objeto de estudio, el tiempo entre finalización de estudios y recogida de datos, el procedimiento de entrevista, el tiempo de duración del trabajo de campo, la tasa de respuesta, la definición operativa de conceptos teóricos diversos o las escalas utilizadas, entre otros aspectos, no son del todo homogéneos. Y aunque es pertinente poner de manifiesto, cuando se dispone de información, la coincidencia o la divergencia de los resultados alcanzados en estudios similares, estas circunstancias obligan a tomar con precaución las comparaciones que se puedan establecer.

Además, y aunque no podemos cuestionar la utilidad de este tipo de estudios hay que enmarcarlos en una coyuntura económico-social determinada y éste, concretamente, se ha realizado en un período de crisis económica mundial al que hay que hacer referencia. Es por ello por lo que hay que darles el valor que tienen en el momento oportuno, pero no podemos olvidar que este tipo de estudios deben ir realizándose periódicamente para, de esta forma, actualizar la información y enmarcarlos en su contexto temporal. Es fundamental además, en este tipo de estudios, contar con la participación del mayor número posible de miembros de la población objetivo para conseguir resultados relevantes.

En relación con esta investigación la conclusión más significativa que podemos sacar es que la Licenciatura en Publicidad y Relaciones Públicas es una carrera con una buena salida laboral, puesto que la mayoría de los egresados estaba trabajando en el momento de realización del estudio y, además, se trata de un trabajo con el que se encuentran satisfechos. Además, los estudiantes que han finalizado la carrera están ocupando un puesto de trabajo que guarda relación con los estudios cursados, por tanto, podemos afirmar que la mayoría de personas que obtiene el título de Publicidad y Relaciones Públicas no sólo busca un trabajo relacionado con su titulación sino que, además, lo encuentra. Son de destacar, también, aspectos positivos como la rápida inserción laboral y la baja tasa de desempleo. Esto nos permite ser optimistas 
de cara a las matriculaciones futuras, puesto que estamos hablando de una titulación que permite conseguir empleo y además un empleo acorde con la formación recibida.

Respecto al tipo de contrato, se aprecia una importante presencia de la temporalidad y aunque lo expuesto en este artículo no lo recoge, de los resultados se desprende una creciente tendencia al trabajo autónomo, ya que empresas se encuentran en una fase de externalización de servicios por lo que los profesionales freelance están en aumento. Sería interesante hacer un estudio con más detalle de este fenómeno.

De manera positiva hemos de entender la valoración de la carrera por parte de los encuestados. Para la mayoría la formación recibida de cara a un futuro desempeño profesional es buena, de hecho, la mayoría volvería a estudiar lo mismo y, además, lo volvería a hacer en la misma institución, dato que podemos considerar muy positivo.

También encontramos una serie de debilidades en dicho estudio, como el hecho de no poder comparar los resultados del mismo con otros trabajos dado su carácter estático así como por la aplicación de diferentes metodologías. Es de destacar la dificultad a la hora de contactar con los sujetos de la muestra lo que reduce la misma de manera significativa. Además, podemos afirmar que los datos analizados se muestran insuficientes para analizar el estado del empleo de nuestros egresados en Publicidad. Para obtener datos concluyentes sería necesario realizar un estudio mínimo anual que nos permitiera relacionar estos datos con los oficiales del INEM.

Para que los estudios de seguimiento de egresados generen la información pertinente, es necesario el compromiso serio de las instituciones bajo estudio, en particular con respecto a la creación de condiciones para la recolección y procesamiento de los datos. Este compromiso debe definirse a mediano plazo a través de la implementación de políticas y de líneas estratégicas a nivel institucional para la instrumentalización y operacionalización de este tipo de estudios. A largo plazo, los estudios de seguimiento requieren ser parte integral del quehacer de los programas académicos como elemento de una cultura de evaluación y mejoramiento institucional.

Debido a que estos estudios permiten el fomento de la cultura de evaluación y el mejoramiento institucional, es necesaria la definición de políticas y estrategias para la realización de los mismos. Es importante asumir la realización de estos estudios como un mecanismo para la rendición de cuentas a la sociedad, como diagnóstico de las capacidades y herramienta para remediar las limitaciones de las universidades.

De forma más positiva podemos entender el hecho de que el presente estudio constituye una valiosa fuente de información para los dirigentes universitarios, puesto que los resultados permiten analizar la validez de los programas de estudio y llevar a cabo, si fuese preciso, los ajustes oportunos. Les sirven, a su vez, para medir el éxito de su institución, en función de los resultados de sus egresados. Eso sí, no podemos concluir sin afirmar que estas estadísticas de empleo, bastante más esperanzadoras que las ofrecidas por las fuentes públicas, deben ser tomadas con cautela. 
En cualquier caso "estos estudios constituyen una alternativa para el autoconocimiento y para la planeación de procesos de mejora y consolidación de las instituciones educativas” (Fresán, 1998).

\section{Bibliografía}

ANECA (2005): El Libro Blanco de Títulos de Grado en Ciencias de la Comunicación, http://www.aneca.es/activin/activin_conver_LLBB.asp.

Barajas, G. (2000): La práctica profesional del Médico Cirujano y Homeópata. Un estudio exploratorio. México: Universidad Autónoma de Tlaxcala.

Blaug, M. (1978): Economics of education. Oxford, England: Pergamon Press.

Cabrera, A. y Weerts, D. (2003): Encuestas a egresados: tres fundamentos conceptuales en el seguimiento de egresados universitarios. Métodos de análisis de la inserción laboral de los universitarios. Salamanca: Universidad de León.

Carnoy, M. (1982): “Economía y Educación”, Revista Educación, n. 40. Vol. III, 4a. época. Consejo Nacional Técnico de la Educación, México, Abril-Junio de 1982.

Flores, P. (2005): Educación superior y desarrollo humano. El caso de tres universidades tecnológicas. México: ANUIES.

Fresán, M. (1998): Los estudios de egresados. Una estrategia para el autoconocimiento y la mejora de las instituciones de educación superior. Esquema básico para estudios de egresados. México: ANUIES

Gleizes, J. (2000): “El capital Humano”, http://multitudes.samizdat.net./article.php3?id_article=312

González, M. A. (1999): Seguimiento de trayectorias escolares. México: Ed. de la asociación nacional de universidades e instituciones de educación superior.

Harbison, F. Y Myers, C. (1964): Education, manpower, and economic growth: Strategies of human resource development. Nueva York: McGraw-Hill.

Ministerio de Educación, Cultura y Deporte (2012): Datos y cifras del Sistema Universitario Español. Curso 2011/2012, https://sede.educacion.gob.es/publiventa/descargas.action?f_codigo=14917\&codigoOpc ion=3

Muñoz, C. (1985): "Aplicación de la teoría económica a la planeación de la educación superior. Reflexiones teóricas y sugerencias metodológicas", ponencia presentada en el II coloquio de pedagogía "La educación hoy", Facultad de filosofía y letras. México: UNAM.

Navarro, M. (1998): Consideraciones teóricas para el estudio de egresados. Esquema básico para el estudio de egresados. México: Ed. Anuies.

Schultz, T. (1968): Valor económico de la educación. México: Uteha. 
Universidad Complutense de Madrid: Inserción Laboral de los titulados de la UCM, http://www.ucm.es /info/portalempleo/serv_estit.htm.

VV. AA. (2001): Libro Blanco. Títulos de grado en Comunicación. Madrid: ANECA, http://www.aneca.es/activin/docs/libroblanco_comunicacion_def.pdf

VV. AA. (2010): Los estudios de comunicación en España: reflexiones en torno al Libro Blanco. Madrid: Icono 14. 